\title{
FIXED POINT THEOREMS FOR ARC-PRESERVING MAPPINGS OF UNIQUELY ARCWISE-CONNECTED CONTINUA
}

\author{
J. B. FUGATE AND LEE MOHLER
}

(Communicated by James E. West)

\begin{abstract}
Let $X$ be a uniquely arcwise-connected continuum and $f: X \rightarrow X$ a map which is arc-preserving (i.e., the image of each arc is an arc or a point). We prove that $f$ has a fixed point.
\end{abstract}

\section{INTRODUCTION}

A continuum $X$ is said to be uniquely arcwise-connected, abbreviated uac, if given any $x, y \in X, x \neq y$, there is a unique arc in $X$, denoted $[x, y]$, whose endpoints are $x$ and $y$. In general these spaces do not have the fixed point property (see [10]), even for monotone and/or open mappings (see [6]). However, planar uac continua have the fixed point property (see [2]) and all uac continua have the fixed point property for homeomorphisms (see [5]). All of the examples in [6] and [10] make use of a common device for producing fixed point free mappings: an arc in the space is mapped over a triod, two legs of which form the arc, and the third leg of which is stretched off to infinity by the given mapping, allowing the "rabbit" to escape.

In section 4 of this paper we show that something like the above is generic for fixed point free mappings of uac continua. Namely, if a self-mapping $f$ of a uac continuum is arc-preserving (i.e., $f(A)$ is an arc or a point for every arc $A$ in the domain of $f$, implying that arcs cannot map over triods), then $f$ must have a fixed point. This result (Theorem 17 below) generalizes the main theorem of [5] and also a theorem attributed to Bellamy (see Corollary 18 below). The proof is based on the proof in [5] and makes use of measure-theoretic techniques. In section 3 below we describe a general setting in which measure techniques can be used to prove fixed point theorems. (See Theorem 10 and the remark following.) In section 2 we prove a lemma (Lemma 4) fundamental to the proof of our fixed point theorem and which yields as a corollary a fixed point theorem for arc-preserving mappings of dendroids over themselves (Theorem 6). We begin with some preliminary definitions and two key lemmas. In what follows, all mappings will be assumed to be continuous.

Definition 1. A simple triod is a union of three arcs (i.e. homeomorphic images of the unit interval $[0,1]$ of real numbers) which meet only in a point $v$, which

Received by the editors November 22, 1993.

1991 Mathematics Subject Classification. Primary 54H25. 
is an endpoint of all three arcs. The arcs are called the legs of the triod, and $v$ is called its vertex. The endpoints of the legs of the triod which are opposite the vertex will be called the endpoints of the triod. It is straightforward to show that if $X$ is a uac continuum and $x, y$ and $z$ are points of $X$ which do not lie together on an arc, then there is a unique simple triod in $X$, whose endpoints are $x, y$ and $z$. This triod will be denoted $T(x, y, z)$.

The proofs of the following two lemmas are a good warmup for reading the rest of the paper.

Lemma 2 (the triod lemma). Let $X$ and $Y$ be uac continua and $f: X \rightarrow Y$ an arc-preserving mapping. If $T \subset X$ is a simple triod with vertex $v$ and $f(T)$ is not an arc, then $f(T)$ is a simple triod with vertex $f(v)$.

Lemma 3 (the arc lemma). Let $X$ and $Y$ be uac continua with $X \subset Y$, and let $f: X \rightarrow Y$ be a fixed point free arc-preserving mapping. Let $I_{0} \subset X$ and $I_{1} \subset Y$ be arcs such that $I_{0} \cup f\left(I_{0}\right) \subset I_{1}$. Let $<$ be one of the two natural orders on $I_{1}$. Suppose that for some $z_{0} \in I_{0}, z_{0}<f\left(z_{0}\right)$. Then $z<f(z)$ for all $z \in I_{0}$.

\section{A FIXED POINT THEOREM FOR DENDROIDS}

Lemma 4. Let $X$ and $Y$ be uac continua and $f: X \rightarrow Y$ a fixed point free arcpreserving mapping such that $X \subset f(X) \subset Y$. Then there is a "reverse sequence" of points $\ldots, x_{-2}, x_{-1}, x_{0}$ in $X$ such that for all $i=\ldots,-2,-1$,

(i) $f\left(x_{i}\right)=x_{i+1}$ and

(ii) $x_{i} \in\left[x_{i-1}, x_{i+1}\right]$.

Proof. Let $z$ be a point in $X$ such that $f(z), f^{2}(z)$ and $f^{3}(z)$ are all defined and all lie in $X$.

Claim 1. There is an $x \in X$ such that $f(x) \in X$ and $f(x) \in\left[x, f^{2}(x)\right]$.

Proof of Claim 1. Suppose first $z, f(z)$ and $f^{2}(z)$ lie together on an arc. If $f(z) \in\left[z, f^{2}(z)\right]$, we are done. If $z \in\left[f(z), f^{2}(z)\right]$, the arc lemma is violated where $I_{0}=[z, f(z)]$ and $I_{1}=f([z, f(z)])$. So suppose $f^{2}(z) \in[z, f(z)]$. Applying the arc lemma again with $I_{0}=\left[f^{2}(z), f(z)\right]$ and $I_{1}=f([z, f(z)])$, we conclude that $f^{3}(z)$ has the same order relation to $f^{2}(z)$ that $f^{2}(z)$ has to $f(z)$, i.e. $f^{2}(z) \in\left[f(z), f^{3}(z)\right]$. So we may take $x=f(z)$.

Finally, suppose that $z, f(z)$ and $f^{2}(z)$ are the endpoints of a triod in $X$ with vertex $v$. Taking $I_{0}=[v, f(z)]$ and $I_{1}=f([z, f(z)])$, the arc lemma implies that $f$ must map $v$ "in the direction of $f^{2}(z)$ " in the arc $I_{1}$. (Consider the behavior of $f$ at $f(z)$.) Moreover, since $v \in f([z, f(z)])$, there must be $v^{\prime} \in[z, f(z)]$ such that $f\left(v^{\prime}\right)=v$. It follows that we can take $x=v^{\prime}$, completing the proof of the claim.

Claim 2. Let $x$ be as in Claim 1, and suppose that for some $n, f(x) f^{2}(x), \ldots$, $f^{n}(x)$ are all elements of $X$. Then for all $i=1,2, \ldots, n$,

$$
f^{i}(x) \in\left[f^{i-1}(x), f^{i+1}(x)\right] .
$$

Proof of Claim 2. This follows by applying the arc lemma inductively with $I_{0}=\left[f^{i-1}(x), f^{i}(x)\right], I_{1}=f\left(\left[f^{i-2}(x), f^{i}(x)\right]\right)$ and $z_{0}=f^{i-1}(x)$, starting with $i=2$. 
We now make our first attempt to define the reverse sequence $\ldots, x_{-2}, x_{-1}$, $x_{0}$ by taking $x_{0}=f(x)$ and $x_{-1}=x$ where $x$ is as in Claim 1. If we are able to keep choosing $x_{-(i+1)}$ 's such that $f\left(x_{-(i+1)}\right)=x_{-i}$ and $x_{-i} \in\left[x_{-(i+1)}, x_{-i+1}\right]$, then we are done. If this process breaks down at some point, $x_{0}$ will have to be redefined. Without loss of generality, we may assume that we are unable to choose $x_{-2}$, i.e., there is no $x^{\prime} \in X$ such that $f\left(x^{\prime}\right)=x$ and $x \in\left[x^{\prime}, f(x)\right]$.

Let $x$ be as in Claim 1, and let $x^{\prime}$ be any preimage of $x$. Then (a) $x \notin$ $\left[x^{\prime}, f(x)\right]$. Also (b) there must be a natural number $n$ such that $f(x), f^{2}(x)$, $\ldots, f^{n-1}(x)$ are all elements of $X$ and $f^{n}(x) \notin\left[x^{\prime}, x\right]$. If this were not the case, then successive applications of Claim 2 , starting with $n=1$, would force $f^{n+1}(x)$ to be defined for every $n$ and the $f^{n}(x)$ 's would converge to a fixed point for $f$ in the arc $\left[x^{\prime}, x\right]$. So let $n \geq 1$ be chosen so that $f^{n-1}(x) \in\left[x^{\prime}, x\right]$ and $f^{n}(x) \notin\left[x^{\prime}, x\right]$. A straightforward application of the arc lemma shows that (c) $x^{\prime} \notin\left[x, f^{n}(x)\right]$. Then (a), (b) and (c) together imply that $x^{\prime}, x$ and $f^{n}(x)$ do not lie together on an arc. So they must be the endpoints of the triod $T\left(x^{\prime}, x, f^{n}(x)\right)$ in $Y$.

Consider the location of the vertex $v$ of $T\left(x^{\prime}, x, f^{n}(x)\right)$ : either $v \in$ $[x, f(x)]$ or $f(x) \in[x, v]$.

Case 1. $v \in[x, f(x)]$ and $v \neq f(x)$. Then $x^{\prime}, x$ and $f(x)$ are the endpoints of a triod with vertex $v$. Let $x^{\prime \prime}$ be any preimage of $x^{\prime}$ under $f$. Then $x^{\prime \prime}, x^{\prime}$ and $x$ cannot lie together on an arc, for such an arc would map over the triod $T\left(x^{\prime}, x, f(x)\right)$. Let $v^{\prime}$ be the vertex of the triod $T\left(x^{\prime \prime}, x^{\prime}, x\right)$. Then by the triod lemma $f\left(v^{\prime}\right)=v$. If $v^{\prime} \in[v, f(x)]$, then $v^{\prime}=v$ and $v$ is a fixed point for $f$. So either $v^{\prime} \in\left[x^{\prime}, v\right]$ or $v^{\prime} \in[x, v]$. However, the latter possibility contradicts the arc lemma: take $I_{0}=\left[x^{\prime}, v^{\prime}\right], I_{1}=f\left(\left[x^{\prime}, x^{\prime \prime}\right]\right)$ and consider the behavior of $f$ at $x^{\prime}$ and $v^{\prime}$. So $v^{\prime} \in\left[x^{\prime}, v\right]$. Next let $x^{\prime \prime \prime}$ be any preimage of $x^{\prime \prime}$. An argument similar to the foregoing will show that $x^{\prime \prime \prime}, x^{\prime \prime}$ and $x^{\prime}$ are the endpoints of a triod with vertex $v^{\prime \prime}$, such that $v^{\prime \prime} \in\left[x^{\prime \prime}, v^{\prime}\right]$ and $f\left(v^{\prime \prime}\right)=v^{\prime}$. Moreover, it is straightforward to check that $v^{\prime} \in\left[v^{\prime \prime}, v\right]$. Let $x_{0}=v, x_{-1}=v^{\prime}$ and $x_{-2}=v^{\prime \prime}$. Continuing inductively in the above manner, we may choose the desired $x_{-i}, i=0,1,2, \ldots$.

Case 2. $f(x) \in[x, v]$. Let $k \leq n-1$ be such that $f^{k}(x) \in[x, v]$ and $f^{k+1}(x) \notin[x, v]$. (Note that by Claim $2 f^{k+1}(x) \in\left[v, f^{n}(x)\right]$.) Applying the arc lemma with $I_{0}=\left[f^{k}(x), v\right], I_{1}=f\left(\left[f^{k}(x), x^{\prime}\right]\right)$ and $z_{0}=f^{k}(x)$, we see that $f$ maps $v$ "away from $x$ " in the arc $f\left(\left[f^{k}(x), x^{\prime}\right]\right) \supset\left[f^{k+1}(x), x\right]$. Therefore $x^{\prime}, x$ and $f(v)$ are the endpoints of a triod with vertex $v$. Let $x^{\prime \prime}$ be any premiage of $x^{\prime}$ under $f$. Then $x^{\prime \prime}, x^{\prime}$ and $x$ cannot lie together on an arc, for such an arc would map over the triod $T\left(x^{\prime}, x, f(v)\right)$. Let $v^{\prime}$ be the vertex of the triod $T\left(x^{\prime \prime}, x^{\prime}, x\right)$. The rest of the proof is as in Case 1.

Definition 5. A dendroid is a uac continuum $D$ with the additional property that given any $x, y \in D,[x, y]$ is the unique subcontinuum of $D$ irreducible with respect to containing both $x$ and $y$.

Theorem 6. Let $D$ be a dendroid, $Y$ a uac continuum and $f: D \rightarrow Y$ an arc-preserving mapping such that $D \subset f(D) \subset Y$. Then $f$ has a fixed point.

Proof. It is straightforward to show $D$ has the following property (see e.g. [1]):

(*) Let $I_{1} \subset I_{2} \subset \cdots$ be a nested sequence of arcs in $D$. Then there is an arc $A$ in $D$ such that $\bigcup_{n=1}^{\infty} I_{n} \subset A$. 
Let $\cdots x_{-2}, x_{-1}, x_{0}$ be a reverse sequence as in Lemma 4, and for each $n$ let $I_{n}=\left[x_{-n}, x_{0}\right]$. Let $A$ be as in $(*)$. Then it is straightforward to show that $x=\lim _{n \rightarrow \infty} x_{-n}$ is a fixed point for $f$.

Remarks. Theorem 6 was proved earlier for finite trees by Fugate and Eberhart (unpublished). Note that the proof of Theorem 6 requires only that $D$ be uac and satisfy $(*)$. It is known (see [4]) that such continua need not be dendroids. So Theorem 6 can be generalized slightly.

Question. Does Theorem 6 hold if $D$ is a uac continuum?

\section{MeAsure AND FiXed POINTS}

The following theorem has been attributed to Krylov and Bogolioubov (see [8], p. 152 for the attribution and proof):

Theorem 7. Let $X$ be a compact metric space and $f: X \rightarrow X$ a continuous function. Then there is a non-negative complete regular Borel measure $\mu$ on $X$ such that $\mu(X)=1$ and for every $\mu$-measurable subset $A$ of $X, \mu(A)=$ $\mu\left(f^{-1}(A)\right)$.

Definition 8. A subset $A$ of a complete separable metric space is said to be analytic if $A$ is the continuous image of a Borel subset of some complete separable metric space.

Lemma 9. Let $X$ be a compact metric space, $f: X \rightarrow X$ a continuous function, $A$ an analytic subset of $X$ and $\mu$ a measure on $X$ as in Theorem 7. Then for every integer $n, f^{n}(A)$ is $\mu$-measurable and $\mu(A) \leq \mu\left(f^{n}(A)\right)$.

Proof. It is well known that each of the sets $f^{n}(A)$ is analytic and therefore $\mu$-measurable. ${ }^{1}$ If $n$ is negative, then by Theorem 7, $\mu(A)=\mu\left(f^{n}(A)\right)$. For $n$ non-negative, we have $A \subset f^{-n}\left(f^{n}(A)\right)$. Therefore, $\mu(A) \leq \mu\left(f^{-n}\left(f^{n}(A)\right)\right)=$ $\mu\left(f^{n}(A)\right)$.

Theorem 10. Let $X$ be a compact metric space and $f: X \rightarrow X$ a continuous function. Then $X$ does not admit a covering by a countable family of analytic sets $A_{i}, i=1,2,3, \ldots$, such that for each $i$, there is an infinite set $Z_{i}$ of integers with the property that the family $\left\{f^{n}\left(A_{i}\right): n \in Z_{i}\right\}$ is disjoint.

Proof. Just suppose that $X$ admitted such a covering. Let $\mu$ be as in Theorem 7. Then, by Lemma 9 , for each $i=1,2,3, \ldots$, and for each $n \in Z_{i}, f^{n}\left(A_{i}\right)$ is $\mu$-measurable and $\mu\left(A_{i}\right) \leq \mu\left(f^{n}\left(A_{i}\right)\right)$. Thus for each $i$,

$$
\sum_{n \in Z_{i}} \mu\left(A_{i}\right) \leq \sum_{n \in Z_{i}} \mu\left(f^{n}\left(A_{i}\right)\right)=\mu\left(\bigcup_{n \in Z_{i}} f^{n}\left(A_{i}\right)\right) \leq \mu(X)=1 .
$$

This clearly implies that $\mu\left(A_{i}\right)=0$ for each $i$. But this contradicts the fact that the $A_{i}$ 's cover $X$ and $\mu(X)=1$.

Remark. In one version of the "dog and rabbit" fixed point argument, the dog and rabbit are a point and its iterates under the map $f$. The dog corners the

\footnotetext{
${ }^{1}$ The authors have been unable to find a good reference for these two results. A proof can be extracted from [3], $\S \S 38,39$. A key element in the proof is the fact that a set is analytic if and only if it can be realized from the closed subsets of $X$ via operation $\mathscr{A}$. It follows immediately that preimages of analytic sets are analytic.
} 
rabbit to produce a fixed point, as in the proof of Theorem 6 . Theorem 10 is something like a dog and rabbit setup. The dog and rabbit are a set $A_{i}$ and its iterates. If the family $\left\{f^{n}\left(A_{i}\right): n \in Z_{i}\right\}$ is disjoint, then the rabbit has escaped. Theorem 10 is saying that you cannot cover a compactum with rabbits that always escape.

\section{A FIXED POINT THEOREM FOR UAC CONTINUA}

Throughout this section we will assume that $X$ is a uac continuum and that $f: X \rightarrow X$ is a fixed point free arc-preserving mapping. A contradiction will be derived, thus proving Theorem 17 below. By a standard argument, we may (and do) assume without loss of generality, that $f$ is a surjection. Thus the hypotheses of Lemma 4 are satisfied. Let $\ldots, x_{-2}, x_{-1}, x_{0}$ be as in Lemma 4. Then every $n=1,2, \ldots$ satisfies the hypotheses of Claim 2 of the proof of that result, with $x=x_{0}$. For each positive integer $n$, let $x_{n}=f^{n}(x)$. Then the set $\bigcup_{n=-\infty}^{\infty}\left[x_{n}, x_{n+1}\right]$ is easily seen to be a "doubly infinite ray", i.e., a oneto-one continuous image of the set $\mathbb{R}$ of all real numbers. We will denote the former set $R$. The sets $\bigcup_{n=0}^{\infty}\left[x_{n}, x_{n+1}\right]$ and $\bigcup_{n=0}^{\infty}\left[x_{-n}, x_{-n-1}\right]$ will be denoted $R^{+}$and $R^{-}$respectively. $R$ inherits a natural order $<$ from $\mathbb{R}$.

Lemma 11. For every $x \in R$

(i) $f(x) \in R$,

(ii) $x<f(x)$, and

(iii) $\lim _{n \rightarrow \infty} f^{n}(x)=\infty$ (i.e., for every integer $m$, there is an integer $n_{0}$ such that $f^{n}(x)>x_{m}$ for all $\left.n>n_{0}\right)$.

Proof. (i) Let $x \in R$ and suppose that $f(x) \notin R$. Then there must be an $n>0$ such that $x_{n} \notin\left[x_{0}, f(x)\right]$. Otherwise $R^{+} \subset\left[x_{0}, f(x)\right]$ and the points $x_{n}, n>0$, would converge to a fixed point for $f$. By similar reasoning, there is an $m<0$ such that $x_{m} \notin\left[f(x), x_{0}\right]$. Since $f(x) \notin R$, it follows that $\left[x_{0}, f(x)\right] \cup\left[x_{m}, x_{0}\right] \cup\left[x_{0}, x_{n}\right]$ is a simple triod, indeed the triod $T\left(x_{m}, f(x), x_{n}\right)$. By making $m$ smaller and $n$ larger if necessary, we may also assume that $x \in\left[x_{m-1}, x_{n-1}\right]$. But then $f$ maps the arc $\left[x_{m-1}, x_{n-1}\right]$ over the triod $T\left(x_{m}, f(x), x_{n}\right)$, a contradiction.

(ii) follows straightforwardly from the arc lemma, since $x_{n} \leq x_{n+1}=f\left(x_{n}\right)$ for every integer $n$.

(iii) follows straightforwardly from the fact that $f$ is fixed point free.

The following lemma is proved by an argument similar to the preceding proof of (i) with $x$ playing the role of $f(x)$ above. The desired $r(x)$ will be the vertex of the triod $T\left(x_{m}, x, x_{n}\right)$.

Lemma 12. For each $x \in X-R$, there is a unique point $r(x) \in R$, such that $[x, r(x)] \cap R=\{r(x)\}$.

Definition 13. Let $x \in X$. If $x \in X-R$, we define $r(x)$ as in the previous lemma. If $x \in R$, we set $r(x)=x$. We will call $r(x)$ the root of $x$. For a set $I \subset R$, we define $A(I)$ to be $\{x \in X: r(x) \in I\} . A(I)$ is the union of all the arcs in $X$ "hanging off" $I$.

The proof of the following lemma is just like the proofs of Lemmas 2.6 and 2.8 in [5].

Lemma 14. If $I \subset R$ is an arc, then $A(I)$ is an analytic set. 
Let $<$ be the natural order on $R$ used in Lemma 11. For $x \in R$, we define $[x, \infty)$ to be $\{y \in R: x \leq y\}$.

Lemma 15. For every $x \in X$, either $r(f(x))=f(r(x))$ or $f(x) \in[r(x), \infty)$. Proof. If $x \in R$, the result is immediate, so we may assume that $x \in X-$ $R$. First suppose that $f(x) \in X-R$. Choose integers $m$ and $n$ such that $x_{m+1}<\min \left\{r(x), r(f(x)\}\right.$ and $\left.x_{n}>\max \{r(x), r(f(x)))\right\}$. Then the triod $T\left(x_{m}, x, x_{n}\right)$ with vertex $r(x)$ maps over the triod $T\left(x_{m+1}, f(x), x_{n+1}\right)$ with vertex $r(f(x))$. So by the triod lemma, $f(r(x))=r(f(x))$.

Now suppose $f(x) \in R$. Let $x^{\prime}$ be any preimage of $x$ under $f$. Then by Lemma 11(i), $x^{\prime} \in X-R$. By the previous paragraph, $f\left(r\left(x^{\prime}\right)\right)=r(x)$. By Lemma 11(ii) this implies that $r\left(x^{\prime}\right)<r(x)$. Finally, consider the location of $f(x) \in R$. If $f(x)<r(x)$, then the arc $\left[x^{\prime}, x\right]$ maps over the triod $T(f(x), x, f(r(x)))$. So we must have $r(x) \leq f(x)$.

Lemma 16. Let $n$ be an integer. Then there is a natural number $m$ such that the family $\left\{f^{-k m}\left(A\left(\left[x_{n-1}, x_{n}\right]\right)\right): k=1,2,3, \ldots\right\}$ is disjoint.

Proof. By Lemma 11 and a straightforward compactness argument, there is a natural number $r$ such that $f^{r}\left(\left[x_{n-1}, x_{n+1}\right]\right) \subset\left[x_{n+1}, \infty\right)$. By Lemma 11(ii), it follows that $f^{r}\left(\left[x_{n-1}, \infty\right)\right) \subset\left[x_{n+1}, \infty\right)$. So by Lemma 15, $f^{r}\left(A\left(\left[x_{n-1}, x_{n+1}\right]\right)\right)$ $\subset\left[x_{n-1}, \infty\right) \cup A\left(\left[x_{n+1}, \infty\right)\right)$. From this, it follows that $f^{2 r}\left(A\left(\left[x_{n-1}, x_{n+1}\right]\right)\right) \subset$ $A\left(\left[x_{n+1}, \infty\right)\right)$. Thus $f^{2 r}\left(A\left(\left[x_{n-1}, x_{n+1}\right]\right)\right) \cap A\left(\left[x_{n-1}, x_{n+1}\right]\right)=\varnothing$. Indeed for all $s \geq 2 r, f^{s}\left(A\left(\left[x_{n-1}, x_{n+1}\right]\right)\right) \cap A\left(\left[x_{n-1}, x_{n+1}\right]\right)=\varnothing$. Taking $m=2 r$, the lemma follows straightforwardly.

Clearly the sets $A\left(\left[x_{n-1}, x_{n+1}\right]\right)$ cover $X$. So Lemmas 14 and 16 contradict Theorem 10. We have proved

Theorem 17. Let $X$ be a uac continuum, and let $f: X \rightarrow X$ be an arc-preserving (continuous) mapping. Then $f$ has a fixed point.

The following result has been attributed to David Bellamy. It is a fixed point theorem for a special class of monotone mappings of uac continua. Recall (see Introduction) that not all monotone maps have fixed points.

Corollary 18. Let $X$ be a uac continuum and $f: X \rightarrow X$ a (continuous) mapping such that for every $x \in X, f^{-1}(x)$ is arcwise-connected. Then $f$ has a fixed point.

Proof. It is not difficult to show that $f$ is arc-preserving.

Remarks. Theorem 17 applies a fortiori to uac continua $X$ with the additional property that if $R$ is any ray in $X$, then $R$ limits onto a set in $X$ with the fixed point property. In [9] this result is claimed for all continuous functions. However, a counterexample is given in [7]. So the result claimed in [9] is partly saved. It can proved without resort to the measure techniques required to prove Theorem 17. It is a straightforward corollary of Lemma 11.

\section{BIBLIOGRAPHY}

1. Karol Borsuk, A theorem on fixed points, Bull. Acad. Polon. Sci. Cl. III 2 (1954), 17-20.

2. Charles Hagopian, Uniquely arcwise connected plane continua have the fixed point property, Trans. Amer. Math. Soc. 248 (1979), 85-104.

3. Kazimierz Kuratowski, Topology, Vol. I, Academic Press, New York, 1966. 
4. Andrzej Lelek and Lee Mohler, On the topology of curves. III, Fund. Math. 71 (1971), 147-160.

5. Lee Mohler, The fixed point property for homeomorphisms of 1-arcwise connected continua, Proc. Amer. Math. Soc. 52 (1975), 451-456.

6. Lee Mohler and Lex G. Oversteegen, Open and monotone fixed point free maps on uniquely arcwise connected continua, Proc. Amer. Math. Soc. 95 (1985), 476-482.

7. Miroslaw Sobolewski, A uniquely arcwise connected continuum without the fixed point property, Bull. Polish Acad. Sci. Math. 34 (1986), 307-313.

8. Peter Walters, An introduction to ergodic theory, Springer-Verlag, New York, 1982.

9. L. E. Ward, A fixed point theorem for chained spaces, Pacific J. Math. 9 (1959), 1273-1278.

10. Gail S. Young, Fixed point theorems for arcwise connected continua, Proc. Amer. Math. Soc. 11 (1960), 880-884.

Department of Mathematics, University of Kentucky, Lexington, Kentucky 405060027

E-mail address: fugate@ms.uky.edu

Department of Mathematics, Saint Martin's College, Lacey, Washington 98503-1297

E-mail address: lmohler@stmartin.wa.com 